POS $\quad \begin{aligned} & \text { PROCEEDINGS } \\ & \text { OF SCIENCE }\end{aligned}$

\title{
One-loop Single Real Emission Contributions to Inclusive Higgs Production at NNNLO
}

\section{W. Kilgore*}

Physics Department, Brookhaven National Laboratory Upton, New York 11973, U.S.A.

E-mail: kilgore@bnl.gov

I discuss the contributions of the one-loop single-real-emission amplitudes, $g g \rightarrow H g, q g \rightarrow H q$, etc. to inclusive Higgs boson production through next-t0-next-to-next-to-leading order in the strong coupling.

Loops and Legs in Quantum Field Theory

27 April 2014 - 02 May 2014

Weimar, Germany

\footnotetext{
* Speaker.
} 


\section{The Discovery of the Higgs Boson}

The most important result from the early runs at the LHC has been the discovery of a 126 $\mathrm{GeV}$ scalar boson that my be the long-sought Higgs boson of the Standard Model. While the measurements of its couplings still have large uncertainties, the gross features look very much like what is expected for the Higgs. The identification of this particle, the determination of whether it is the SM Higgs boson, a component of an extended symmetry breaking sector or even an impostor, is the most important task in our field today.

In order to make this identification, we need to measure the "Higgs" as thoroughly as possible. We need to measure the mass, the width, the cross section, and the couplings. We need to look for more "Higgs" bosons and for other new particles that might be connected to the "Higgs". These are difficult tasks and will require a great deal of data at the full energy of the LHC.

One of the simplest observables associated with the Higgs, the cross section, is actually difficult to take advantage of. The reason for this is that the theoretical uncertainty in the cross section is very large. This is the case even though the cross section has been computed at next-to-next-toleading order [1 -3] and resummed to next-to-next-to-leading log accuracy [4, 5].

One of the main sources of uncertainty in the gluon fusion cross section comes from the scale dependence of the partonic cross section. This uncertainty can be addressed by computing the cross section at higher order in $\alpha_{s}$. This means performing the calculation at next-to-next-to-nextto-leading order (NNNLO).

\section{Inclusive Higgs Production at NNNLO}

There are many contributions to inclusive Higgs production at NNNLO, and all have been computed in the threshold approximation: Virtual contributions through three loops [6-9], oneloop single real emission squared [10,11], two-loop single real emission [12], one-loop double real emission [12,13] and triple real emission at tree level [14]. Virtual corrections only contribute at threshold, so that term is known completely, as an expansion in the dimensional parameter $\varepsilon$. The full kinematic dependence of the squared one-loop single real emission contrbution, the subject of this talk, has also been computed as an expansion in $\varepsilon[10,11]$. Among the reasons that full kinematic dependence of this term can be computed are that the one-loop amplitudes are known in closed analytic form and because the phase space element for single-real emission is particularly simple.

\subsection{The Heavy Top Effective Theory}

The Higgs boson couples to mass, therefore it does not couple directly to massage gauge bosons like gluons and photons. Instead, such particles have indirect couplings to the Higgs through heavy particle loops. The interaction between gluons and the Higgs is dominated by the top quark, while photons couple through both top and $W$ boson loops. Because the top pair production threshold is much heavier than the Higgs, one can form an effective Lagrangian for Higgs - gluon interactions by integrating out the top quark [15 - 17] for the Higgs-gluon interaction:

$$
\mathscr{L}_{\text {eff }}=-\frac{H}{4 v} C_{1}\left(\alpha_{s}\right) G_{\mu v}^{a} G^{a \mu v},
$$


. where $C_{1}$ is the Wilson coefficient, known to $\mathscr{O}\left(\alpha_{s}^{4}\right)$ [18-21], and $G_{\mu \nu}^{a}$ is the gluon field strength tensor. Using the effective Lagrangian greatly simplifies calculations as it transforms massive top quark loops in to point-like vertices.

\subsection{One-loop Single Real Emission}

The amplitude for single-real emission, computed at any order, can be written in terms of a small number of gauge invariant tensors. There are four gauge invariant tensor structures for $\mathrm{Hggg}$ amplitudes [22, 23] and only two structures [23] for $H q \bar{q} g$ amplitudes,

$$
\begin{aligned}
& \mathscr{M}\left(H ; g_{1}, g_{2}, g_{3}\right)=\frac{g}{v} C_{1}\left(\alpha_{s}\right) f^{i j k} \varepsilon_{1 \mu}^{i} \varepsilon_{2 v}^{j} \varepsilon_{3 \rho}^{k} \sum_{n=0}^{3} A_{n} \mathscr{Y}_{n}^{\mu v \rho}, \\
& \mathscr{M}(H ; g, q, \bar{q})=i \frac{g}{v} C_{1}\left(\alpha_{s}\right)\left(T^{g}\right)_{j}^{\bar{l}} \varepsilon_{\mu}\left(p_{g}\right)\left(B_{1} \mathscr{X}_{1}^{\mu}+B_{2} \mathscr{X}_{2}^{\mu}\right) .
\end{aligned}
$$

The coefficient of each tensor has an expansion in $\alpha_{s}$ of the form

$$
A_{i}=A_{i}^{(0)}+\left(\frac{\alpha_{s}}{\pi}\right) A_{i}^{(1)}+\left(\frac{\alpha_{s}}{\pi}\right)^{2} A_{i}^{(2)}+\ldots,
$$

and the same for the $B_{i}$. I have computed the amplitudes in the following manner: the Feynman diagrams were generated using QGRAF [24]; they were contracted with the projectors onto the gauge-invariant tensors and the Feynman rules were implemented using a FORM [25] program. For the one-loop amplitudes, the resulting expressions were reduced to loop master integrals with the program REDUZE2 [26]. The reduced expressions were put back into the FORM program and the master integrals were evaluated to produce the final expressions.

There are two loop master integrals that appear in these amplitudes, the bubble and the singlemass box. Both are known in closed analytic form for arbitrary kinematics. In a frame where $s_{12}>0, s_{23}, s_{31}<0$

$$
\begin{aligned}
\mathscr{I}_{2}^{(1)}\left(Q^{2}\right) & =\frac{i c_{\Gamma}}{\varepsilon(1-2 \varepsilon)}\left(\frac{\mu^{2}}{-Q^{2}}\right)^{\varepsilon} \\
\mathscr{I}_{4}^{(1)}\left(s_{12}, s_{23} ; M_{H}^{2}\right) & =\frac{2 i c_{\Gamma}}{s_{12} s_{23}} \frac{1}{\varepsilon^{2}}\left[\left(\frac{\mu^{2}}{-s_{12}}\right)^{\varepsilon}{ }_{2} F_{1}\left(1,-\varepsilon ; 1-\varepsilon ;-\frac{s_{31}}{s_{23}}\right)\right. \\
& +\left(\frac{\mu^{2}}{-s_{23}}\right)^{\varepsilon}{ }_{2} F_{1}\left(1,-\varepsilon ; 1-\varepsilon ;-\frac{s_{31}}{s_{12}}\right) \\
& \left.-\left(\frac{\mu^{2}}{-M_{H}^{2}}\right)^{\varepsilon}{ }_{2} F_{1}\left(1,-\varepsilon ; 1-\varepsilon ;-\frac{M_{H}^{2} s_{31}}{s_{12} s_{23}}\right)\right] \\
\mathscr{I}_{4}^{(1)}\left(s_{23}, s_{31} ; M_{H}^{2}\right) & =\frac{2 i c_{\Gamma}}{s_{23} s_{31}} \frac{1}{\varepsilon^{2}}\left[\left(\frac{\mu^{2}}{-s_{12}}\right)^{-\varepsilon}\left(\frac{\mu^{2}}{-s_{23}}\right)^{\varepsilon}\left(\frac{\mu^{2}}{-s_{31}}\right)^{\varepsilon} \Gamma(1-\varepsilon) \Gamma(1+\varepsilon)\right. \\
& +\left(\frac{\mu^{2}}{-s_{23}}\right)^{\varepsilon}\left(1-{ }_{2} F_{1}\left(1, \varepsilon ; 1+\varepsilon ;-\frac{s_{31}}{s_{12}}\right)\right) \\
& +\left(\frac{\mu^{2}}{-s_{31}}\right)^{\varepsilon}\left(1-{ }_{2} F_{1}\left(1, \varepsilon ; 1+\varepsilon ;-\frac{s_{23}}{s_{12}}\right)\right) \\
& \left.-\left(\frac{\mu^{2}}{-M_{H}^{2}}\right)^{\varepsilon}\left(1-{ }_{2} F_{1}\left(1, \varepsilon ; 1+\varepsilon ;-\frac{s_{23} s_{31}}{s_{12} M_{H}^{2}}\right)\right)\right]
\end{aligned}
$$


where

$$
c_{\Gamma}=\frac{\Gamma(1+\varepsilon) \Gamma^{2}(1-\varepsilon)}{(4 \pi)^{2-\varepsilon} \Gamma(1-2 \varepsilon)} .
$$

\subsection{Squared amplitudes and Phase Space Integration}

The partonic cross section is computed by squaring the amplitudes and integrating over phase space

$$
\sigma=\frac{1}{2 s_{12}} d(L I P S) \frac{1}{\mathbb{S}} \sum_{\text {spin/color }}|\mathscr{M}|^{2},
$$

where the factor of $1 /\left(2 s_{12}\right)$ is the flux factor, $d(L I P S)$ represents Lorentz invariant phase space and the factor $1 / \mathbb{S}$ represents the averaging over initial state spins and colors. The element of Lorentz invariant phase space is

$$
d(L I P S)=\frac{1}{8 \pi}\left(\frac{4 \pi \mu^{2}}{s_{12}}\right)^{\varepsilon} \frac{\left(s_{23} s_{31}\right)^{\varepsilon}}{\Gamma(1-\varepsilon)} d s_{23}
$$

Defining $s_{12}=\hat{s}$ to be the parton CM energy squared, I introduce the dimensionless parameters $x=M_{H}^{2} / \hat{s}, \bar{x}=1-x$, and $y=\frac{1}{2}\left(1-\cos \theta^{*}\right), \bar{y}=1-y$, where $\theta^{*}$ is the scattering angle in the CM frame,

$$
\begin{array}{ll}
s_{12}=\hat{s}, & M_{H}^{2}=x \hat{s}, \\
s_{23}=\bar{x} y \hat{s}, & s_{31}=\bar{x} \bar{y} \hat{s} .
\end{array}
$$

In terms of these variables, the element of phase space is

$$
d(L I P S)=\frac{1}{8 \pi}\left(\frac{4 \pi^{2} \mu^{2}}{\hat{s}}\right)^{\varepsilon} \frac{1}{\Gamma(1-\varepsilon)} \bar{x}^{1-2 \varepsilon} y^{-\varepsilon} \bar{y}^{-\varepsilon} d y .
$$

$\bar{x}$ is the threshold parameter, and is a measure of excess or kinetic energy in the scattering process, beyond that which is needed to produce a Higgs boson at rest. The kinematically available region in $x$ and $y$ space is $M_{H}^{2} / s<x<1$ and $0<y<1$, where $s$ is the hadronic (not partonic) CM energy. Clearly, $0<\bar{x}<1-M_{H}^{2} / s$ and $0<\bar{y}<1$.

\subsection{Phase Space Master Integrals}

The resulting expression for the partonic cross section consists of a large number of phase space integrals, often with complicated integrands involving the products of two hypergoemtric functions. To simplify the integration, I employ an integration-by-parts style reduction on the phase space integrals. Under the assumption that any term in the integrand can be expressed in the form $f(\bar{x}) y^{\alpha} \bar{y}^{\beta}$, where $\alpha$ and $\beta$ are not negative integers (which is ensured by dimensional regularization), I can use the fact that

$$
\int_{0}^{1} d y \frac{d}{d y} f(\bar{x}) y^{\alpha} \bar{y}^{\beta}=0
$$

to derive relations among various phase space integrals. Eventually, I am able to reduce the problem to that of solving for 24 phase space master integrals. 
I compute the phase space master integrals by performing an extended threshold expansion of some 120 terms for each master integral. I then map the expansions onto a set of harmonic polylogarithms and thereby obtain the results for the master integrals in closed analytic form.

Finally, I substitute the values of the master integrals into the full expression to obtain the squared one-loop single real emission contribution to inclusive Higgs production cross section at NNNLO. I find complete analytic agreement with Ref. [10]. The full result is too lengthy to report here, but can be obtained from the supplementary material attached to the journal article at http://link.aps.org/supplemental/10.1103/PhysRevD.89.073008.

\section{Conclusions}

I have computed the contributions of one-loop single-real-emission amplitudes to inclusive Higgs boson production at NNNLO. Though a complicated calculation, this is but a portion of the full NNNLO result. I have computed this contribution as an extended threshold expansion, obtaining enough terms to invert the series and determine the closed functional form through order $\varepsilon^{1}$.

Acknowledgments: This research was supported by the U.S. Department of Energy under Contract No. DE-AC02-98CH10886.

\section{References}

[1] R. V. Harlander and W. B. Kilgore, Next-to-next-to-leading order Higgs production at hadron colliders, Phys. Rev. Lett. 88 (2002) 201801, [hep-ph/0201206].

[2] C. Anastasiou and K. Melnikov, Higgs boson production at hadron colliders in NNLO QCD, Nucl. Phys. B646 (2002) 220-256, [hep-ph/ 0207004 ] .

[3] V. Ravindran, J. Smith, and W. L. Van Neerven, Next-to-leading order QCD corrections to differential distributions of Higgs boson production in hadron hadron collisions, Nucl. Phys. $\mathbf{B 6 3 4}$ (2002) 247-290, [hep-ph/0201114].

[4] S. Catani, D. de Florian, M. Grazzini, and P. Nason, Soft-gluon resummation for Higgs boson production at hadron colliders, JHEP 07 (2003) 028, [ hep-ph/ 0306211 ] .

[5] S. Moch and A. Vogt, Higher-order soft corrections to lepton pair and Higgs boson production, Phys.Lett. B631 (2005) 48-57, [hep-ph/ 0508265 ] .

[6] R. Lee, A. Smirnov, and V. Smirnov, Analytic Results for Massless Three-Loop Form Factors, JHEP 1004 (2010) 020, [1001.2887].

[7] P. Baikov, K. Chetyrkin, A. Smirnov, V. Smirnov, and M. Steinhauser, Quark and gluon form factors to three loops, Phys.Rev.Lett. 102 (2009) 212002, [0 902.3519 ] .

[8] T. Gehrmann, E. Glover, T. Huber, N. Ikizlerli, and C. Studerus, Calculation of the quark and gluon form factors to three loops in QCD, JHEP 1006 (2010) 094, [1004.3653] .

[9] T. Gehrmann, E. Glover, T. Huber, N. Ikizlerli, and C. Studerus, The quark and gluon form factors to three loops in QCD through to $O\left(\varepsilon^{2}\right), J H E P 1011$ (2010) 102, [1010.4478].

[10] C. Anastasiou, C. Duhr, F. Dulat, F. Herzog, and B. Mistlberger, Real-virtual contributions to the inclusive Higgs cross-section at $N^{3}$ LO, JHEP 1312 (2013) 088, [1311.1425]. 
[11] W. B. Kilgore, One-Loop Single-Real-Emission Contributions to $p p \rightarrow H+X$ at Next-to-Next-to-Next-to-Leading Order, Phys.Rev. D89 (2014) 073008, [1312 .1296] .

[12] C. Anastasiou, C. Duhr, F. Dulat, E. Furlan, T. Gehrmann, et al., Higgs boson gluon-fusion production at threshold in N3LO QCD, [1403.4616].

[13] Y. Li, A. von Manteuffel, R. M. Schabinger, and H. X. Zhu, $N^{3} L O$ Higgs and Drell-Yan production at threshold: the one-loop two-emission contribution, [1404.5839].

[14] C. Anastasiou, C. Duhr, F. Dulat, and B. Mistlberger, Soft triple-real radiation for Higgs production at N3LO, JHEP 1307 (2013) 003, [ 1302 . 4379] .

[15] A. I. Vainshtein, M. B. Voloshin, V. I. Zakharov, and M. A. Shifman, Low-energy theorems for Higgs boson couplings to photons, Yad. Fiz. 30 (1979) 1368. [Sov. J. Nucl. Phys. 30, 711 (1979)].

[16] M. B. Voloshin, Once again about the role of gluonic mechanism in interaction of light Higgs boson with hadrons, Yad. Fiz. 44 (1986) 738. [Sov. J. Nucl. Phys. 44, 478 (1986)].

[17] A. I. Vainshtein, V. I. Zakharov, and M. A. Shifman, Higgs particles, Usp. Fiz. Nauk 131 (1980) 537. [Sov. Phys. Usp. 23, 429 (1980)].

[18] K. Chetyrkin, B. A. Kniehl, and M. Steinhauser, Decoupling relations to $\mathscr{O} \alpha_{s}^{3}$ and their connection to low-energy theorems, Nucl.Phys. B510 (1998) 61-87, [hep-ph/9708255].

[19] K. G. Chetyrkin, B. A. Kniehl, and M. Steinhauser, Hadronic Higgs decay to order $\alpha_{s}^{4}$, Phys. Rev. Lett. 79 (1997) 353-356, [hep-ph/9705240 ].

[20] Y. Schroder and M. Steinhauser, Four-loop decoupling relations for the strong coupling, JHEP 0601 (2006) 051, [hep-ph/0512058].

[21] K. Chetyrkin, J. H. Kuhn, and C. Sturm, QCD decoupling at four loops, Nucl.Phys. B744 (2006) 121-135, [hep-ph/0512060].

[22] R. K. Ellis, I. Hinchliffe, M. Soldate, and J. J. van der Bij, Higgs decay to $\tau^{+} \tau^{-}$: A possible signature of intermediate mass Higgs bosons at the SSC, Nucl. Phys. B297 (1988) 221.

[23] T. Gehrmann, M. Jaquier, E. Glover, and A. Koukoutsakis, Two-Loop QCD Corrections to the Helicity Amplitudes for $H \rightarrow 3$ partons, JHEP 1202 (2012) 056, [1112 . 3554 ] .

[24] P. Nogueira, Automatic Feynman graph generation, J. Comput. Phys. 105 (1993) 279-289.

[25] J. Vermaseren, New features of FORM, [math-ph/0010025].

[26] A. von Manteuffel and C. Studerus, Reduze 2 - Distributed Feynman Integral Reduction, [1201.4330]. 